\title{
OCUPACIÓN DE LAS MUJERES EN EL MEDIO RURAL. DATOS PARA UN DEBATE
}

\author{
Alicia Langreo Navarro $^{a}$ *, Tomás García Azcárate ${ }^{\text {b }}$ \\ a Directora de Sabora, S.L. E-mail: alangreonavarro@gmail.com $\underline{\text { b}}$ Vice-Director del Instituto de \\ Economía, Geografía y Demografía (IEGD-CSIC) e investigador asociado del CEIGRAM, Madrid, E- \\ mail: tomasgarciaazcarate@gmail.com."
}

Resumen: Uno de los problemas de la España Vaciada es la falta de actividad económica de las mujeres, históricamente con menos tasa de actividad y mayor paro. Analizamos su ocupación y evolución en los municipios de menos de 20.000 habitantes Utilizamos la única fuente disponible, el Censo de la Población (último disponible 2011). El análisis se desagrega según sectores económicos y subsectores en su caso, así como por tipo de trabajo. Para algunos capítulos se desagrega según dimensión del municipio y según lugar de nacimiento en España o fuera. La fecha de la realización del último Censo deja fuera del análisis la mayor parte del efecto de las crisis económicas iniciadas en 2008 y la actual, pero el conocimiento de lo que sucedió entonces y cuáles fueron los sectores de éxito en la ocupación femenina serán fundamental para el análisis de las crisis posteriores. Entre 2001 y 2011 mejoró el equilibrio entre la ocupación de hombres y mujeres en España y en el medio rural, en buena parte debido al crecimiento de la ocupación vinculada al desarrollo del estado del bienestar (educación, sanidad, cuidados a las personas) y de la presencia de mujeres la mejora y modernización de los sistemas productivos.

Palabras Claves:_Medio rural; empleo; mujeres

\section{Introducción y metodología}

Esta comunicación se ubica en el trabajo que los autores vienen desarrollando sobre la actividad en la España vaciada. Aborda la actividad de las mujeres en el medio rural en términos laborales-profesionales. Se centra en el análisis de la ocupación de las mujeres en la primera década del siglo XXI. La fuente utilizada para la información estadística recogida en todos los cuadros es el Censo de la Población de los años 2001 y 2011 (el próximo, de 2021 debería estar disponible en 2022), la única fuente que ofrece datos municipales según estratos de dimensión poblacional, sector de actividad, posición profesional, tramos de edad, etc.

Fue un periodo económico expansivo, frenado por la crisis de 2008. Apenas se recogen los efectos de las dos crisis del siglo XXI, pero el comportamiento en esos años da pistas para el futuro y facilita ajustar la orientación de las políticas públicas. Los resultados obtenidos se han contrastado con diversos estudios, aunque la limitación de esta comunicación no permite reseñarlos.

Las definiciones del Censo de la población son las mismas que las de la Encuesta de Población Activa (EPA). Se ha optado por utilizar la población ocupada en vez de la población activa, ya que la falta de oportunidades de trabajo conlleva la bajada de la población activa. Esta fuente no mide el volumen de trabajo anual, sino la ocupación en el momento de la toma de datos, por lo tanto no recoge bien en trabajo estacional o temporal escaso.

Consideramos rurales los municipios de menos de 20.000 habitantes, como primera aproximación. Dado que la variable dimensional se mostrado muy significativa, se ha realizado el análisis según estrato de dimensión.

\section{Resultados}

Entre 2001 y 2011 la población española aumentó en un 14\%, mientras en los municipios menores de 20.000 habitantes sólo un 4\% (5,27\% hombres y 2,55\% mujeres) y en los inferiores a 2.000 habitantes bajó casi un $9 \%$ (Cuadro 1)

\section{Cuadro 1}

\begin{tabular}{|c|c|c|c|c|c|c|c|c|c|}
\hline \multicolumn{10}{|c|}{ Evolución de la población total $2001 / 2011$} \\
\hline & España & $<101 \mathrm{hb}$ & $\begin{array}{l}101 \mathrm{a} \\
500 \mathrm{hb}\end{array}$ & \begin{tabular}{|l|}
$501 \mathrm{a}$ \\
$1.000 \mathrm{hb}$ \\
\end{tabular} & $\begin{array}{l}1.001 \mathrm{a} \\
2.000 \mathrm{hb}\end{array}$ & $\begin{array}{l}2.001 \mathrm{a} \\
5.000 \mathrm{hb}\end{array}$ & \begin{tabular}{|l|}
$5.001 \mathrm{a}$ \\
$10.000 \mathrm{~h}$ \\
\end{tabular} & \begin{tabular}{|l|}
$10001 \mathrm{a}$ \\
$20000 \mathrm{hb}$ \\
\end{tabular} & $<20000$ \\
\hline Total & $14,02 \%$ & $12,41 \%$ & $-6,08 \%$ & $-6,12 \%$ & $-8,61 \%$ & $-0,48 \%$ & $10,09 \%$ & $9,79 \%$ & $4,10 \%$ \\
\hline Hombres & $14,99 \%$ & $17,62 \%$ & $-3,58 \%$ & $-4,37 \%$ & $-7,06 \%$ & $1,04 \%$ & $11,37 \%$ & $10,31 \%$ & $5,27 \%$ \\
\hline Mujeres & $13,09 \%$ & $6,29 \%$ & $-8,79 \%$ & $-7,96 \%$ & $-10,20 \%$ & $-2,02 \%$ & $8,81 \%$ & $8,13 \%$ & $2,55 \%$ \\
\hline
\end{tabular}


En el año 2011 el porcentaje de ocupados en España era del 37,6\% (el 35,77\% en los municipios rurales); entre los hombres el 41,6\% en España y el 40,8\% en los municipios rurales; entre las mujeres, el 33,7\% para España y el 30,6\% en los municipios rurales (Cuadro 2).

\section{Cuadro 2}

\begin{tabular}{|l|r|r|r|r|r|r|r|r|r|}
\hline \multicolumn{7}{|c|}{2011 . Porcentaje de ocupados sobre población total } \\
\hline Habitantes & $<101 \mathrm{Hb}$ & 101 a 500 & 501 a 1000 & 1001 a 2000 & 2001 a 5000 & 5001 a 10000 & $10000-200000$ & & \\
\hline Sexo & & & & & & & & Menores 20000 & España \\
\hline Total & $33,84 \%$ & $34,42 \%$ & $34,07 \%$ & $34,76 \%$ & $35,16 \%$ & $36,27 \%$ & $36,47 \%$ & $35,77 \%$ & $37,61 \%$ \\
\hline Hombres & $41,26 \%$ & $40,83 \%$ & $39,86 \%$ & $40,01 \%$ & $40,41 \%$ & $41,17 \%$ & $41,09 \%$ & $4,80 \%$ & $41,62 \%$ \\
\hline Mujeres & $24,20 \%$ & $27,08 \%$ & $27,78 \%$ & $29,20 \%$ & $29,74 \%$ & $31,27 \%$ & $31,85 \%$ & $30,59 \%$ & $33,69 \%$ \\
\hline
\end{tabular}

\begin{tabular}{|c|c|c|c|c|c|c|c|c|c|}
\hline \multicolumn{10}{|c|}{2001 Porcentaje de ocupados sobre población total } \\
\hline Habitantes & $<101 \mathrm{Hb}$ & 101 a 500 & 501 a 1000 & 1001 a 2000 & 2001 a 5000 & 5001 a 10000 & $10000-200000$ & & \\
\hline Sexo & & & & & & & & Menores 20000 & España \\
\hline Total & $34,77 \%$ & $34,92 \%$ & $35,21 \%$ & $35,97 \%$ & $37,39 \%$ & $38,90 \%$ & $39,70 \%$ & $38,11 \%$ & $39,98 \%$ \\
\hline Hombres & $47,60 \%$ & $47,01 \%$ & $46,92 \%$ & $47,54 \%$ & $48,93 \%$ & $50,55 \%$ & $50,91 \%$ & $49,61 \%$ & $50,41 \%$ \\
\hline Mujeres & $19,72 \%$ & $21,85 \%$ & $22,99 \%$ & $24,15 \%$ & $25,81 \%$ & $27,31 \%$ & $28,63 \%$ & $26,57 \%$ & $29,95 \%$ \\
\hline
\end{tabular}

En 2011 la diferencia entre la cuota de ocupados entre hombres y mujeres en España era casi de 8 puntos y en el medio rural de más de 10. Estas cifras habían mejorado mucho desde 2001, cuando era casi de más de 20 puntos (España) y 24 en el medio rural (Cuadro 3).

Cuadro 3

\begin{tabular}{|c|c|c|c|c|c|c|c|c|c|}
\hline \multicolumn{10}{|c|}{ Personas ocupadas en municipios menores 20000 hb. Censo de la Población 2011} \\
\hline Habitantes & $<101 \mathrm{Hb}$ & 101 a 500 & 501 a 1000 & 1001 a 2000 & 2001 a 5000 & 5001 a 10000 & $10000-200000$ & \multirow{2}{*}{$\begin{array}{l}\text { Total municipios } \\
<20000 \mathrm{hb}\end{array}$} & \multirow[b]{2}{*}{ España } \\
\hline Sexo & & & & & & & & & \\
\hline Total & 20999 & 249431 & 280505 & 512957 & 1180241 & 1360477 & 1855134 & 5459744 & 16329713 \\
\hline Hombres & 15520 & 174439 & 190899 & 342609 & 773315 & 882006 & 1181506 & 3560294 & 10089395 \\
\hline Mujeres & 5479 & 74992 & 89606 & 170348 & 406926 & 478471 & 673628 & 1899450 & 6240318 \\
\hline$\%$ mujeres & $26,09 \%$ & $30,07 \%$ & $31,94 \%$ & $33,21 \%$ & $34,48 \%$ & $35,17 \%$ & $36,31 \%$ & $34,79 \%$ & $38,21 \%$ \\
\hline
\end{tabular}

La situación era mucho peor en los municipios con menos de 5.000 habitantes y empeoraba según disminuía la población. Entre 2001 y 2011, el número de ocupados en España aumentó un 7,26\% para toda la población, cayó algo más de un 5\% entre los hombres y subió más de un 27\% en mujeres. En los municipios rurales, el número de ocupados disminuyó un 2,31\%: el de hombres cayó el 13,43\% mientras que el de mujeres subió un $18 \%$.

Encontramos una diferencia positiva a favor de las mujeres en todos los estratos de población en los municipios menores de 20.000 habitantes, mayor en los comprendidos entre 5.000 y 20.000 habitantes, aunque el crecimiento de las mujeres ocupadas ha sido inferior en los municipios rurales que en la media nacional.

El aumento de mujeres ocupadas en el medio rural responde al desarrollo de actividades propiciatorias de su integración, intensa en los últimos años del siglo XX y en la primera década del XXI. 
Cuadro 4

\begin{tabular}{|c|c|c|c|c|c|c|}
\hline \multicolumn{7}{|c|}{ Porcentaje de ocupados según rama de actividad y sexo. 2011} \\
\hline & \multicolumn{3}{|c|}{ España } & \multicolumn{3}{|c|}{ Municipos $<20000$ habitantes } \\
\hline & $\begin{array}{l}\text { Población } \\
\text { total }\end{array}$ & $\begin{array}{l}\text { Hombre } \\
\mathrm{s}\end{array}$ & Mujeres & $\begin{array}{l}\text { Población } \\
\text { total }\end{array}$ & $\begin{array}{l}\text { Hombre } \\
s\end{array}$ & Mujeres \\
\hline Agricultura ganadería silvicultura y pesca & $4,46 \%$ & $5,75 \%$ & $2,90 \%$ & $9,21 \%$ & $11,53 \%$ & $6,04 \%$ \\
\hline Industrias extractivas & $0,26 \%$ & $0,39 \%$ & $0,11 \%$ & $0,42 \%$ & $0,63 \%$ & $0,14 \%$ \\
\hline I manufacturera & $12,10 \%$ & $15,78 \%$ & $7,66 \%$ & $15,29 \%$ & $18,71 \%$ & $10,60 \%$ \\
\hline Suministro Electricidad, gas, vapor y aire acondicionado & $0,72 \%$ & $1,02 \%$ & $0,35 \%$ & $0,70 \%$ & $0,99 \%$ & $0,30 \%$ \\
\hline Suministro agua, saneamiento, gestión residuos y descontaminación & $0,62 \%$ & $0,88 \%$ & $0,29 \%$ & $0,65 \%$ & $0,91 \%$ & $0,31 \%$ \\
\hline Construcción & $7,73 \%$ & $12,30 \%$ & $2,22 \%$ & $10,13 \%$ & $15,71 \%$ & $2,49 \%$ \\
\hline Comercio al mayor y menor, reparación vehículos de motos y bicis & $14,29 \%$ & $13,11 \%$ & $15,70 \%$ & $14,30 \%$ & $12,40 \%$ & $16,90 \%$ \\
\hline Transporte y almacenamiento & $4,78 \%$ & $6,96 \%$ & $2,15 \%$ & $4,60 \%$ & $6,45 \%$ & $2,06 \%$ \\
\hline Hostelería & $6,97 \%$ & $6,16 \%$ & $7,95 \%$ & $6,84 \%$ & $5,28 \%$ & $8,98 \%$ \\
\hline Información y comunicaciones & $3,27 \%$ & $3,86 \%$ & $2,55 \%$ & $1,88 \%$ & $2,14 \%$ & $1,52 \%$ \\
\hline Actividades financieras y seguros & $2,91 \%$ & $2,79 \%$ & $3,06 \%$ & $1,96 \%$ & $1,73 \%$ & $2,28 \%$ \\
\hline Actividades inmobiliarias & $0,51 \%$ & $0,43 \%$ & $0,61 \%$ & $0,36 \%$ & $0,29 \%$ & $0,46 \%$ \\
\hline Actividades profesionales científicas y técnicas & $4,88 \%$ & $4,87 \%$ & $4,89 \%$ & $3,23 \%$ & $3,01 \%$ & $3,54 \%$ \\
\hline Actividades administrativas y servicios auxiliares & $3,86 \%$ & $2,89 \%$ & $5,03 \%$ & $3,09 \%$ & $2,20 \%$ & $4,32 \%$ \\
\hline Administración pública y defensa, seguridad social obligatoria & $7,87 \%$ & $8,20 \%$ & $7,47 \%$ & $6,84 \%$ & $7,07 \%$ & $6,52 \%$ \\
\hline Educación & $8,10 \%$ & $4,94 \%$ & $11,90 \%$ & $6,91 \%$ & $3,79 \%$ & $11,18 \%$ \\
\hline Actividades sanitarias y de servicios sociales & $8,71 \%$ & $4,30 \%$ & $14,03 \%$ & $7,10 \%$ & $3,00 \%$ & $12,70 \%$ \\
\hline Actividades artísticas recreativas y de entretenimiento & $1,64 \%$ & $1,68 \%$ & $1,59 \%$ & $1,30 \%$ & $1,28 \%$ & $1,33 \%$ \\
\hline Otros servicios & $3,79 \%$ & $2,95 \%$ & $4,80 \%$ & $3,37 \%$ & $2,39 \%$ & $4,71 \%$ \\
\hline $\begin{array}{l}\text { Actividades en los hogares como empleadores y productores de bienes y } \\
\text { servicios }\end{array}$ & $2,41 \%$ & $0,63 \%$ & $4,56 \%$ & $1,73 \%$ & $0,41 \%$ & $3,53 \%$ \\
\hline Actividades de organizaciones extraterritoriales & $0,13 \%$ & $0,12 \%$ & $0,15 \%$ & $0,09 \%$ & $0,08 \%$ & $0,09 \%$ \\
\hline Total & $100,00 \%$ & $\begin{array}{r}100,00 \\
\%\end{array}$ & $\begin{array}{r}100,00 \\
\%\end{array}$ & $100,00 \%$ & $\begin{array}{r}100,01 \\
\%\end{array}$ & $\begin{array}{r}100,00 \\
\%\end{array}$ \\
\hline
\end{tabular}

Principales rasgos en cuanto al sector de ocupación en los rurales en 2011 (Cuadros 4 y 5):

- Sólo el 6\% de las mujeres ocupadas (11,5\% de los hombres) se dedicaban a la agricultura, ganadería, silvicultura o pesca: de más del $14 \%$ en los municipios más pequeños al $4 \%$ en los comprendidos entre 10.000 y 20.000 habitantes.

- La dedicación a la industria manufacturera suponía a más del 10\% de las mujeres ocupadas, cifra inferior a la de los hombres y por encima de la media nacional. La ocupación según dimensión poblacional oscilaba entre el $8,5 \%$ y el $17,76 \%$.

- El comercio aportaba casi el 17\% de las ocupadas, por encima de la media nacional, oscilando entre casi el $10 \%$ de los municipios pequeños al casi $18 \%$ de los mayores.

- La hostelería reunía a casi el $9 \%$ de las ocupadas, por encima de la media española y por encima del porcentaje de hombres en el mismo estrato.

- En la Administración pública trabajaban más mujeres que en la agricultura.

- Más del $11 \%$ de las mujeres ocupadas se dedicaban a la educación, cerca de la media nacional.

- Las actividades sanitarias y de servicios sociales alcanzaban el 12,7\%, cerca de la media nacional.

\section{Cuadro 5}

\begin{tabular}{|c|c|c|c|c|c|c|c|}
\hline \multicolumn{8}{|c|}{ Porcentaje mujeres ocupadas según actividad y dimensión del municipio 2011} \\
\hline & $\begin{array}{l}\text { Menos } \\
\text { de } \\
101 \mathrm{hb}\end{array}$ & $\begin{array}{l}\text { De } 101 \text { a } \\
500 \mathrm{hb}\end{array}$ & $\begin{array}{c}\text { De } 501 \mathrm{a} \\
1.000 \mathrm{hb}\end{array}$ & $\begin{array}{l}\text { De } 1.001 \mathrm{a} \\
2.000 \mathrm{~h}\end{array}$ & $\begin{array}{l}\text { De } 2.001 \\
5.000\end{array}$ & $\begin{array}{l}\text { De } 5.001 \mathrm{a} \\
10.000 \mathrm{~h}\end{array}$ & $\begin{array}{l}\text { De } \\
10.001 \\
\text { a } 20.000\end{array}$ \\
\hline Agricultura ganadería silvicultura y pesca & $14,83 \%$ & $10,94 \%$ & $8,82 \%$ & $8,42 \%$ & $7,92 \%$ & $5,38 \%$ & $4,00 \%$ \\
\hline Industrias extractivas & $0,14 \%$ & $0,11 \%$ & $0,21 \%$ & $0,22 \%$ & $0,13 \%$ & $0,09 \%$ & $0,15 \%$ \\
\hline I manufacturera & $8,46 \%$ & $9,56 \%$ & $10,21 \%$ & $10,76 \%$ & $11,45 \%$ & $10,95 \%$ & $10,00 \%$ \\
\hline Suministro Electricidad, gas, vapor y aire acondicionado & $0,14 \%$ & $0,22 \%$ & $0,29 \%$ & $0,31 \%$ & $0,31 \%$ & $0,31 \%$ & $0,29 \%$ \\
\hline Suministro agua, saneamiento, gestión residuos y descontaminación & $0,35 \%$ & $0,21 \%$ & $0,25 \%$ & $0,29 \%$ & $0,32 \%$ & $0,30 \%$ & $0,33 \%$ \\
\hline Construcción & $2,87 \%$ & $2,57 \%$ & $2,33 \%$ & $2,63 \%$ & $2,52 \%$ & $2,55 \%$ & $2,40 \%$ \\
\hline
\end{tabular}




\begin{tabular}{|c|c|c|c|c|c|c|c|}
\hline Comercio al mayor y menor, reparación vehículos de motos y bicis & $9,72 \%$ & $12,68 \%$ & $14,60 \%$ & $15,38 \%$ & $16,50 \%$ & $17,59 \%$ & $17,76 \%$ \\
\hline Transporte y almacenamiento & $1,96 \%$ & $2,02 \%$ & $2,17 \%$ & $1,92 \%$ & $2,05 \%$ & $2,06 \%$ & $2,10 \%$ \\
\hline Hostelería & $13,01 \%$ & $12,10 \%$ & $10,55 \%$ & $9,82 \%$ & $8,58 \%$ & $8,12 \%$ & $9,08 \%$ \\
\hline Información y comunicaciones & $1,26 \%$ & $1,14 \%$ & $1,13 \%$ & $1,28 \%$ & $1,33 \%$ & $1,60 \%$ & $1,72 \%$ \\
\hline Actividades financieras y seguros & $2,17 \%$ & $1,70 \%$ & $1,96 \%$ & $1,95 \%$ & $2,12 \%$ & $2,36 \%$ & $2,48 \%$ \\
\hline Actividades inmobiliarias & $0,21 \%$ & $0,32 \%$ & $0,30 \%$ & $0,34 \%$ & $0,35 \%$ & $0,48 \%$ & $0,58 \%$ \\
\hline Actividades profesionales científicas y técnicas & $2,38 \%$ & $2,72 \%$ & $2,81 \%$ & $2,76 \%$ & $3,15 \%$ & $3,85 \%$ & $3,89 \%$ \\
\hline Actividades administrativas y servicios auxiliares & $3,57 \%$ & $3,85 \%$ & $4,04 \%$ & $4,14 \%$ & $4,21 \%$ & $4,24 \%$ & $4,57 \%$ \\
\hline Administración pública y defensa, seguridad social obligatoria & $8,74 \%$ & $8,06 \%$ & $7,83 \%$ & $7,17 \%$ & $6,55 \%$ & $6,23 \%$ & $6,23 \%$ \\
\hline Educación & $8,53 \%$ & $9,62 \%$ & $9,92 \%$ & $10,20 \%$ & $10,71 \%$ & $11,48 \%$ & $11,80 \%$ \\
\hline Actividades sanitarias y de servicios sociales & $12,94 \%$ & $13,24 \%$ & $13,52 \%$ & $13,18 \%$ & $12,69 \%$ & $12,69 \%$ & $12,46 \%$ \\
\hline Actividades artísticas recreativas y de entretenimiento & $1,26 \%$ & $1,23 \%$ & $1,15 \%$ & $1,28 \%$ & $1,24 \%$ & $1,41 \%$ & $1,36 \%$ \\
\hline Otros servicios & $3,15 \%$ & $3,68 \%$ & $4,35 \%$ & $4,16 \%$ & $4,48 \%$ & $4,85 \%$ & $5,03 \%$ \\
\hline $\begin{array}{l}\text { Actividades en los hogares como empleadores y productores de bienes y } \\
\text { servicios }\end{array}$ & $4,27 \%$ & $3,95 \%$ & $3,49 \%$ & $3,69 \%$ & $3,33 \%$ & $3,34 \%$ & $3,70 \%$ \\
\hline Actividades de organizaciones extraterritoriales & $0,07 \%$ & $0,10 \%$ & $0,11 \%$ & $0,09 \%$ & $0,07 \%$ & $0,12 \%$ & $0,08 \%$ \\
\hline Total & & & & & & & \\
\hline
\end{tabular}

La ocupación de mujeres cambió entre 2001 y 2011:

- La ocupación agricultura, ganadería, silvicultura y pesca cayó más del $28 \%$.

- La ocupación en industria manufacturera cayó más del $24 \%$.

- La ocupación en hostelería subió más del $18 \%$, y comercio, en torno al $7 \%$.

- En la Administración la ocupación subió más del 9\%.

- Sobre todo subió en el epígrafe de educación (más del 57\%) y actividades sanitarias, veterinarias y de asistencia social (más del 72\%).

\section{Conclusiones}

Entre 2001 y 2011, mejoró sensiblemente la ocupación de las mujeres en el medio rural, aproximándose a la de los hombres, mientras caía su dedicación a la agricultura. La mejora se registró especialmente en actividades ligadas a los servicios a las personas y al estado del bienestar (sanidad, educación, etc.), además de la hostelería y el comercio. Destaca el apartado de técnicos y profesionales de apoyo, de gran importancia en la modernización y cualificación la estructura productiva.

Este comportamiento es coherente con que el emprendimiento de las mujeres que se concentró en sectores vinculados a la extensión de labores tradicionales y en aquellos en los que previamente habían sido asalariadas, como comercio u hostelería (Cruces Roldán y Palenzuela Chamorro, 2006, Saborá 1999 y 2006).

Sólo en agricultura se encontró una gran diferencia en la ocupación según tramos de edad: frente a una media del 6\%, sólo el 3,7\% en las menores de 30 años, entre de 30 a 50 años menos del 5\%, y en los tramos de mayor edad $10 \%$ entre 50 y 65 años y más del $16 \%$ por encima.

La mejora de la ocupación en áreas de actividad que requieren cualificación es del máximo interés ya que supone una inflexión en la huida de las mujeres más cualificadas (Sampedro Gallego, 1991; Camarero y Sampedro, 2008).

\section{Bibliografía}

Camarero, L.; Sampedro, R. (2008): ¿Por qué se van las mujeres? El continuum de movilidad como hipótesis explicativa de la masculinización rural Revista Española de Investigaciones Sociológicas (Reis) $124,73-105$

Cruces Roldán, C.; Palenzuela Chamorro, P. (2006): Emprendedoras rurales en Andalucía. Posibilidades y límites de sus estrategias Revista Española de Estudios Agrosociales y Pesqueros, 211, 239-305 Saborá (1999):"NNuevos Yacimientos de Empleo. Orientaciones para su activación por mujeres en el ámbito rural". Realizado para el Instituto de la Mujer.

Saborá (2006): La respuesta de las mujeres rurales a los programas operativos de desarrollo rural del MAPA para el periodo 2000-2006. Realizado para el MAPA.

Sampedro Gallego, R. (1991): El Mercado de trabajo en el medio rural: una aproximación a través del género Política y Sociedad 8, 25-33 